\title{
Urinary Incontinence in Elderly Women: Urodynamic Evaluation
}

\author{
Ananias C. Diokno, MD, Thelma J. Wells, PhD, RN, + and Carol A. Brink, MPH, RNt
}

The objective of this study is to characterize urinary incontinence observed in elderly women and to assess the importance of various parameters used to evaluate urinary incontinence. Two hundred consecutive, ambulatory, outpatient, incontinent women 55 years of age and over who were seen at the Continence Program Clinic and completed a medical and urodynamic protocol are included in this paper. After a thorough medical history and a complete physical examination, urodynamic tests were performed. The urodynamic results showed that $77 \%$ of incontinent women had an incompetent urethra. Twenty-five percent had a hyperactive bladder, $8 \%$ had "other" types, and $7 \%$ had a normal study. Comparison of the clinical diagnosis with the actual urodynamic diag- nosis for stress incontinence revealed a $78 \%$ accuracy and only a $6 \%$ false negative. In contrast, a similar comparison for urge incontinence found only $44 \%$ accurate and $45 \%$ false negative. Analysis of the urodynamic tests revealed that the simple provocative full-bladder stress test was as effective as the radiographic or electronic pressure measurement in detecting incompetent urethra producing stress urinary incontinence. Provocative upright cystometry was helpful in uncovering $33 \%$ of hyperactive bladders not provoked in the supine position. Complex urodynamic tests should be reserved for unexplained incontinence or when symptomatology is complex. J Am Geriatr Soc 35:940-946, 1987
I ncontinence of urine is a major problem of the elderly. The impact of this problem extends far beyond its physical, psychological, and social effects on the elderly themselves to the economics of the affected individual, their family, and the community as a whole. A recently published study in the United States reported an over-all prevalence of $30 \%$ in a community population 60 years of age and older. ${ }^{3}$

Please see related editorial in the September issue, JAGS 35:880-882.

From the *William Beaumont Hospital, Royal Oak, Michigan, and the University of Michigan School of Medicine, Ann Arbor, Michigan; the HUniversity of Rochester, School of Nursing, Rochester, New York.

The study was funded by the National Institute on Aging (1R01 AG03542).

Portions of this paper were presented at the XIIIth International Congress of Gerontology, New York City, New York, July 1985.

Address correspondence and reprint requests to Dr. Wells, University of Rochester, School of Nursing, 601 Elmwood Avenue, Rochester, NY 14642.
European surveys indicate a prevalence of 1.6 to $49 \%$ among noninstitutionalized persons. ${ }^{1,2}$

Women are much more affected by urinary incontinence than men. Thomas et al. reported a prevalence of urinary incontinence of $11.6 \%$ in women and $6.9 \%$ in men 65 years of age and over, and $8.5 \%$ in women and $1.6 \%$ in men 15 to 65 years of age. ${ }^{4}$ Yarnell and St. Leger reported 17 and $11 \%$ prevalence of urinary incontinence in women and men 65 years of age and over, respectively. ${ }^{5}$ And, in an American sample, Diokno et al. found $38 \%$ of women and $19 \%$ of men 60 years of age and older incontinent. ${ }^{3}$

There is an unquestionable need to learn more about urinary incontinence in the elderly. This paper will focus on the characteristics of urinary incontinence comparing it with clinical diagnosis and discussing the value of the diagnostic methodology used to evaluate this problem among ambulatory older women. Further study detail, including recruitment procedures, sample characteristics, and clinical findings will be found in the companion paper. ${ }^{6}$ 


\section{MATERIALS AND METHODS}

Two hundred consecutive ambulatory outpatient women 55 years of age and over who consulted the Continence Program Clinic because of urinary incontinence and who completed a prospective evaluation protocol are included in this report. Urinalysis was performed, and urine culture was obtained when necessary. Urinary infections were treated appropriately. A thorough medical history, which included a detailed analysis of incontinence patterns and a complete physical examination including a pelvic and rectal examination, was obtained. A clinical diagnosis of stress urinary incontinence was made when the patient described loss of urine while straining, coughing, bending over, and/or provided objective evidence of stress loss during the physical examination. The loss of urine was clinically considered urge incontinence when loss of urine followed an immediate toilet need and nocturia of three or more times was reported. Incontinence was considered mixed when the clinical criteria for both stress incontinence and urge incontinence were present. Any other symptom of urine loss not classified as stress or urge were then considered in the "other" category which is generally the overflow decompensated bladder.

Urodynamic evaluation included uroflowmetry, residual urine determination, combined cystometry, sphincter electromyography and rectal manometry, simultaneous bladder and urethral pressure measurements, stress cystourethrography, and provocative full-bladder stress testing. Uroflowmetry was the initial test done; however, when the patient was unable to void or the voided volume was less than 150 $\mathrm{mL}$, the test was repeated at the end of the study. For pressure measurements, an $8 \mathrm{Fr}$. Millar double transducer catheter was used to monitor the bladder and urethral pressures. Intrarectal pressure was monitored using an esophageal membrane catheter, and a coaxial needle electrode was used to detect and monitor the electromyographic activity of the external sphincter. Recording of all activities was made using the DISA 6 channel urovideo recorder, except for 14 patients when a Life Tech Urodynamic unit was used.

Cystometry was done initially in the supine position using a $25 \%$ Hypaque solution at a flow rate of $100 \mathrm{~mL} /$ minute. Residual urine volume was always measured prior to cystometry. The capacity was considered reached when the patient complained of extreme urgency or an intense desire to void, suprapubic pain, or with the onset of a strong uninhibited contraction. When no uncontrolled contraction was observed at capacity, the patient was asked to cough five times to provoke uninhibited contractions. If none were provoked, the patient was placed in the upright position; the patient was again asked to cough five times in order to further provoke the bladder to contract involuntarily. An uninhibited contraction was defined in accordance with the International Continence Society's definition. ${ }^{7}$

The dynamic bladder and urethral profilometry was monitored continuously during the five separate coughing maneuvers while in the supine and upright positions. During the five consecutive coughing maneuvers, the urethral pressure was subtracted from the bladder pressure to determine any evidence of incontinence, which is defined as bladder pressure equal to or greater than the midurethral pressure.

Cystography was performed when the bladder was at capacity and the patient was in the upright lateral position to demonstrate incontinence radiographically and to evaluate the position and mobility of the bladder and urethra. Spot cystograms were taken while the patient was at rest and again while the patient was straining. Stress incontinence was documented when contrast was observed inside the urethra on spot cystourethrography during the exertional maneuver.

Finally, with all the catheters removed, provocative full-bladder stress testing was carried out. The patient was asked to strain and cough, and incontinence was observed and quantified. The observer was alerted to discriminate between urine loss during and after coughing, since loss of urine occurring after stress would suggest detrusor contraction provoked by exertion.

The final urodynamic diagnosis included detrusor hyperactivity, urethral incompetency, mixed detrusor hyperactivity and urethral incompetency, normal bladder and urethral function, and other categories. Detrusor hyperactivity was defined as the presence of uninhibited or uncontrolled contraction $\geq 10 \mathrm{~cm}$ water on cystometry during filling. This urodynamic diagnosis is considered the mechanism for urge incontinence.

Urethral incompetency was established when urinary leakage was demonstrated while straining or coughing during stress cystography or during provocative stress testing with a full bladder or when the bladder pressure equaled or exceeded urethral pressure during the coughing maneuvers in the supine and/or upright positions. Urethral incompetency was considered the mechanism for stress type of urinary incontinence in our subjects.

When the urodynamic findings demonstrated both the presence of detrusor hyperactivity and urethral incompetency, the diagnosis was called mixed or complex type. This urodynamic diagnosis is considered equivalent to the clinical diagnosis of mixed incontinence.

Any other abnormalities documented during the urodynamic evaluation that did not fit the previous 
TABLE 1. SELECTED URODYNAMIC EVALUATION FINDINGS

\begin{tabular}{|c|c|c|c|c|}
\hline Test & $\mathbf{N}$ & Range & Mean & SD \\
\hline \multicolumn{5}{|l|}{ Uroflowmetry } \\
\hline Peak flow rate* & 119 & $6-50$ & 22 & 9 \\
\hline \multicolumn{5}{|l|}{ Cystometry } \\
\hline Residual urine & 199 & $0-500$ & 26 & 61 \\
\hline Capacity & 200 & $100-650$ & 377 & 124 \\
\hline \multicolumn{5}{|l|}{ Urethral proflilometry } \\
\hline Maximum urethral pressure & 197 & $16-130$ & 58.4 & 21.6 \\
\hline Sphincter electromyography & 195 & 163 (normal) & 32 (abnormal) & \\
\hline
\end{tabular}

*Only for preinstrumented subjects whose volume voided ranged from 150 to $500 \mathrm{~mL}$.

diagnoses were grouped in the "other" category. Most of the abnormalities in this group included the hypotonic or decompensated bladder with or without neurogenic dysfunction. When no abnormalities were found, the patient was categorized as normal.

All patients were discharged on an antibacterial preparation of either trimethoprim-sulfamethoxazole or nitrofurantoin for three days. They were seen in the Continence Program Clinic for follow-up two to three weeks after the urodynamic study.

\section{RESULTS}

The ages of these 200 patients ranged from 55 to 90 years of age (mean, 68.5 years; SD, 8.6 years). There were 78 patients in the 55 to 64 years of age range, 70 in the 65 to 74 years of age range, and 52 in the 75 years of age and over range.

Table 1 reports selected findings from the urodynamic evaluation. Only preinstrumented subjects whose volume voided ranged from 150 to $500 \mathrm{~mL}$ are reported for peak flow rate. The mean age for this subset was 68 years of age (SD, 8.6 years); peak flow rate was not significantly different for the three age groups (ANOVA, $P>.05$ ). The mean peak flow of 22 $\mathrm{mL} /$ second is within the reported acceptable range for this population. ${ }^{8}$ Following uroflowmetry, subjects (N $=199$ ) were catheterized for residual in preparation for cystometric testing. Thirty-five subjects had no residual while 144 ranged from 1 to $50 \mathrm{~mL}, 12$ from 51 to $100 \mathrm{~mL}$, and eight from 101 to $500 \mathrm{~mL}$. The residual amount varied significantly with age group (ANOVA, $P=.05$ ). Women aged 55 to 64 years of age had a mean residual of $13 \mathrm{~mL}$ in contrast to a mean of $35 \mathrm{~mL}$ for those aged 65 to 74 years of age, and a mean of $34 \mathrm{~mL}$ for those aged 75 to 90 years of age. The mean bladder capacity at cystometrics ( 377 $\mathrm{mL}$; SD, 124) was within normal limits but wide variance occurred in range of capacity. Capacity was significantly related to age group (ANOVA, $P<.05$ ) with women 75 to 90 years of age having a mean capacity of $342 \mathrm{~mL}$ in contrast to $384 \mathrm{ml}$ for women aged 55 to 64 years of age, and $401 \mathrm{~mL}$ for women aged 64 to 74 years of age. Twenty percent of the women were found to have abnormal sphincter needle myography; age group was not significantly associated. The mean maximum urethral pressure (MUP) was 58 (SD, 22), which is within the reported range for normal women over 64 years of age. ${ }^{8}$ The MUP was significantly related to age group (ANOVA, $P<.05$ ) with women aged 75 to 90 years of age having an average MUP of 48 in contrast to 64 for women aged 55 to 64 years of age, and 60 for women aged 65 to 74 years of age.

The clinical impressions based upon the patients' description of urine loss and the findings on physical

TABLE 2. CLINICAL IMPRESSIONS BASED UPON THE PATIENTS' DESCRIPTION OF URINE LOSS AND THE FINDINGS ON PHYSICAL EXAMINATION

\begin{tabular}{|c|c|c|c|c|c|c|}
\hline \multirow[b]{2}{*}{ Clinical Diagnosis } & \multirow[b]{2}{*}{$\mathbf{N}$} & \multicolumn{5}{|c|}{ Urodynamic Diagnosis } \\
\hline & & $\begin{array}{c}\text { Urethral } \\
\text { Incompetency }\end{array}$ & $\begin{array}{c}\text { Detrusor } \\
\text { Hyperactivity }\end{array}$ & Mixed & Normal & Other \\
\hline Stress & 132 & 92 & 3 & 15 & 12 & $10^{*}$ \\
\hline Urge & 8 & 3 & 2 & 2 & 0 & 1 \\
\hline Mixed & 53 & 24 & 8 & 14 & 2 & $5^{*}$ \\
\hline Other & 7 & 2 & 1 & 2 & $\mathbf{0}$ & 2 \\
\hline Total & 200 & 121 & 14 & 33 & 14 & 18 \\
\hline
\end{tabular}

*Two subjects were not able to complete urodynamic testing because of strong, uninhibited bladder contractions during cystometrics. Since incompetent urethra could not be ruled out in these cases, they were classified as gross urge and are in the "other" urodynamic category with one each in stress and mixed by clinical diagnosis. However, when appropriate, these two cases are also discussed within the hyperactive bladder category. 
examination were stress in 132 , urge in eight, mixed in 53, and other in seven (Table 2). The urodynamic evaluations revealed solely urethral incompetency in 121 , detrusor hyperactivity in 14 , mixed in 33 , normal in 14 , and other in 18.

The clinical diagnosis agreed perfectly with the urodynamic diagnosis for $110(55 \%)$ of the patients. There were 49 patients for whom one diagnosis included both urge and stress symptoms (mixed), while the other diagnosis indicated a pure type. The two diagnoses were diametrically opposed for only six patients (three diagnosed as stress clinically and urge urodynamically and three diagnosed as urge clinically and stress urodynamically). The clinical and urodynamic diagnosis showed significant agreement $(K=$ $.20 ; \mathrm{p}<.01)$.

There were a total of 185 patients with a clinical diagnosis of stress or exertional incontinence as the only or mixed diagnosis. One hundred forty-two (78\%) of these patients were confirmed by urodynamics to have urethral incompetency or mixed diagnosis. Of the eight patients clinically considered to have only urge incontinence, only half were confirmed to have detrusor hyperactivity (two pure urge, two mixed).

There were 18 patients classified as having a urodynamic diagnosis of "other." Most of these patients predominantly had only a large bladder capacity ( $\geq$ $500 \mathrm{cc}$ ) or a decompensated bladder with an overflow type of urinary incontinence. Most of these patients had presented with either stress or stress-urge symptomatology. Also included in this group were two patients who were not able to complete the urodynamic testing because of strong uninhibited bladder contractions.

There were 14 patients who were found to have no abnormalities on urodynamic investigation. Twelve of these were determined clinically to have pure stress loss and two were considered mixed loss.

Urethral incompetency, either alone or in combination, was established in 154 patients. Of these, 121 patients $(79 \%)$ had no other urodynamic diagnosis in contrast to 33 patients $(21 \%)$ who had mixed diagnosis of detrusor hyperactivity and urethral incompetency.

The diagnosis of urethral incompetency was established on the basis of urine loss observed during static stress cystography in 63 patients, full-bladder stress testing in 122 patients and differential bladder and urethral pressure measurements in 114 of 154 patients. In 104 (68\%) patients, two or more tests were positive for urethral incompetency. The number of vaginally delivered children in those without prior bladder surgery was not significantly different among the urodynamic diagnostic groups $(P>.05)$. However, on physical examination those with a urodynamic diagnosis of urethral incompetency were more likely to have severe cystocele $\left(\chi^{2}=6.55 ; P<.05\right)$, and/or severe rectocele $\left(\chi^{2}=10.55 ; P<.01\right)$.

Of the 49 patients with detrusor hyperactivity, 14 patients had detrusor hyperactivity only, 33 had mixed incontinence, and two were classified as having detrusor hyperactivity with incompetent urethra not ruled out. Provocative upright cystometry was found pos-

TABLE 3. SELECTED CLINICAL DATA BY URODYNAMIC DIAGNOSIS

\begin{tabular}{|c|c|c|c|c|c|c|}
\hline \multirow[b]{2}{*}{ Clinical Data* } & \multicolumn{5}{|c|}{ Urodynamic Diagnosis } & \multirow[b]{2}{*}{$\begin{array}{c}\text { Number of } \\
\text { Subjects } \\
200\end{array}$} \\
\hline & $\begin{array}{c}\text { Urethral } \\
\text { Incompetency } \\
\mathbf{1 2 1}\end{array}$ & $\begin{array}{c}\text { Mixed } \\
33\end{array}$ & $\begin{array}{c}\text { Detrusor } \\
\text { Hyperactivity } \\
14\end{array}$ & $\begin{array}{c}\text { Normal } \\
14\end{array}$ & $\begin{array}{c}\text { Other } \\
18\end{array}$ & \\
\hline \multicolumn{7}{|l|}{ History } \\
\hline Yes position loss & $62 \%$ & $17 \%$ & $6 \%$ & $8 \%$ & $7 \%$ & 143 \\
\hline Yes cough loss & $66 \%$ & $16 \%$ & $5 \%$ & $7 \%$ & $6 \%$ & 140 \\
\hline Yes sneeze loss & $64 \%$ & $16 \%$ & $5 \%$ & $7 \%$ & $8 \%$ & 135 \\
\hline Yes urge loss & $59 \%$ & $19 \%$ & $8 \%$ & $6 \%$ & $8 \%$ & 155 \\
\hline Nocturia three or more & $46 \%$ & $25 \%$ & $16 \%$ & $4 \%$ & $9 \%$ & 69 \\
\hline Day frequency nine or more & $47 \%$ & $23 \%$ & $12 \%$ & $8 \%$ & $10 \%$ & 96 \\
\hline \multicolumn{7}{|l|}{ Examinationt } \\
\hline Positive Standing Stress & $61 \%$ & $26 \%$ & $2 \%$ & $4 \%$ & $7 \%$ & 57 \\
\hline Positive Lying Stress & $71 \%$ & $18 \%$ & 0 & $7 \%$ & $7 \%$ & 28 \\
\hline \multicolumn{7}{|l|}{ Diary $\ddagger$} \\
\hline Nocturia three or more & $57 \%$ & $23 \%$ & $11 \%$ & $4 \%$ & $5 \%$ & 56 \\
\hline Day Frequency nine or more & $51 \%$ & $28 \%$ & $11 \%$ & $5 \%$ & $5 \%$ & 57 \\
\hline
\end{tabular}

*Percents add to $100 \%$ in each row (except for rounding effects).

tStress testing was done in an office setting without prior instrumented bladder filling; 129 had the standing test, 197 the lying.

$¥$ Acceptable diaries were completed by 184 subjects. 
itive in provoking uninhibited detrusor contraction in $16(33 \%)$ of 49 patients. The rest were detected on supine cystometry only.

Night toileting frequency varied significantly with the urodynamic diagnosis (ANOVA; $P<.01$ ). Those with only detrusor hyperactivity recalled an average of four night toilet visits in contrast to two for all other diagnostic groups. However, such toileting on selfmonitored diary records $(\mathrm{N}=179)$ was not significantly different for urodynamic diagnostic groups $(P$ $>.05$ ). None of the chronic illnesses studies were significantly associated with urodynamic diagnostic groups.

Table 3 displays selected clinical data by urodynamic diagnosis. Although a majority of those subjects having urethral incompetency on urodynamic evaluation answered positively to stress loss questions on history and demonstrated stress urine loss on office examination (without instrumented bladder filling), from 2 to $10 \%$ of those without urethral incompetency also were positive on these classic stress incontinence clinical variables. In terms of classic clinical urge (detrusor hyperactivity) symptoms, 59\% of those reporting loss of urine after an immediate urge to toilet were found to have only urethral incompetency in urodynamic evaluation. Nocturnal frequency, selected at three or more times as an additional detrusor hyperactivity clinical criteria, was reported in 69 subjects of whom $58 \%$ were not found to have detrusor hyperactivity. Diary records reflected that $65 \%$ of those with nocturia three or more times were not found to have detrusor hyperactivity. Day frequency of nine or more by history and diary was distributed across urodynamic diagnoses with the largest percentage characteristic of those with only urethral incompetency.

\section{DISCUSSION}

The major focus of this study has been characterization of urinary incontinence frequently seen in help seeking ambulatory elderly women living in the community. Another objective has been to assess the value of the various parameters in diagnosing this problem.

The presence of detrusor hyperactivity in only $25 \%$ of our women is definitely less than the 39 to $75 \%$ reported in previous studies with older females. ${ }^{9-12}$ The discrepancy extends to the diagnosis of urethral incompetency as our series discovered $77 \%$, while others have reported only 5 to $45 \%^{10-12}$ and Castleden et al., did not even report a single case. ${ }^{9}$ The discrepancy between these findings can be attributed to the characteristics of the clinic population being studied since neither ours nor the four other studies are prevalence research. All our patients were ambulatory, noninstitutionalized, and able to largely com- plete urodynamic testing. Only $26 \%$ were 75 years of age or older, and all were in relatively good health. The study by Castleden et al. sampled mixed inpatients and outpatients'; others have not clearly described their sample's health status. ${ }^{10-12}$

Another reason for the discrepancy is either measurement technique variance or lack of tests to identify urethral incompetency. The Castleden et al. study did passive urethral profilometry only and did not attempt to diagnose urethral type incontinence with any specific test. Hilton and Stanton, on the other hand, used video cystourethrography in only 30 of 100 women and the dual sensor for urethral closure pressure testing in only 22 . Eastwood and Warrell had limited diagnostic tests and Ouslander et al. neither tested all subjects with urodynamics nor reported criteria for a stress incontinence diagnosis. In our study subjects received all the tests in a systematic way.

There is a major discrepancy between the types of urinary incontinence obtained from history and physical examination and that of the urodynamic evaluation, a finding supported by previous investigators. ${ }^{9-13}$ The major differences are experienced mostly in those patients clinically determined to be mixed (urge and stress) or pure urge urinary incontinence.

When the symptomatology is stress incontinence the sensitivity of clinical prediction is good. One hundred and eighty-five subjects gave positive responses to one or more probes about stress urine loss and/or demonstrated urine loss on examination (132 as stress only, 53 in combination with urge). Of these, $78 \%$ were found to have stress incontinence by urodynamic evaluation. Six percent actually had urge incontinence only, but perhaps elicited bladder contractions with increased abdominal pressure before urethral incompetency could be demonstrated. Eight percent were classified as "other" by urodynamics and typically presented with an overflow incontinence consistent with decompensated bladder. The $8 \%$ normals may have stress incontinence, but at urodynamic evaluation either did not replicate bladder capacity or adequate abdominal pressure needed to leak or anxiety induced by testing elicited physiological tightening of their sphincter. In general, the stress incontinence clinical criteria demonstrated only a $22 \%$ false-positive response. Most of those subjects would have been treated incorrectly based on clinical findings alone.

Comparing urodynamic diagnosis with clinical shows that 154 subjects had stress incontinence. Of those, $6 \%$ either denied stress loss by history and/or physical examination failed to elicit it, to represent the falsenegative side to clinical evaluation. Half of these had detrusor hyperactivity, the dominance of which could have masked awareness of true stress loss. 
Sixty-one subjects reported nocturia three or more times per night and loss of urine immediately after a toileting urge to be classified as urge incontinence clinically (urge only eight, mixed with stress 53 ). Of these, only $44 \%$ were found to have urge incontinence on urodynamic evaluation. Most (44\%) were found to have stress incontinence only. A sensory urgency is common in stress incontinence and could have elicited both the reported nocturnal frequency and, with position change related to toilet travel, an urge type leakage. Thus, the clinical criteria for urge incontinence had a 57\% false-positive response; these $35 \mathrm{sub}$ jects would have been treated incorrectly without urodynamic evaluation.

Of course it is possible that some false-positive clinical urge subjects did have urge incontinence, but their concentrated effort during urodynamic evaluation or failure to replicate eliciting mechanisms limited the accuracy of such evaluation.

Considering false negatives for the urge clinical criteria, 49 subjects demonstrated urge incontinence on urodynamic evaluation. Of these, $22(45 \%)$ had denied the combination of urge-related urine loss and nocturia three or more times on history.

Cystometry is still the only test available to document the presence of hyperactive bladder. Simple supine fast fill cystometry $(100 \mathrm{~mL} /$ minute) identified detrusor hyperactivity in $67 \%$ of our patients. Provocative upright cystometry was helpful in uncovering hyperactivity in an additional $33 \%$.

There were three tests used in this protocol to identify urethral incontinence. These tests included simultaneous measurements of bladder and urethral pressures at rest and coughing, cystography at rest and straining, and provocative full-bladder stress testing. The result of this study showed that the provocative full-bladder stress testing $(80 \%)$ is the most sensitive in identifying urethral incontinence. The electronic differential bladder-urethral pressure measurement $(73 \%)$ is nearly as sensitive as the provocative testing. The differences in the sensitivity of these tests can be explained by the fact that each test was conducted separately rather than concomitantly; therefore, factors operating during each test may be somewhat different. Since no one test is perfect or superior to the other, it is recommended that if provocative full-bladder testing is negative in eliciting incontinence, the other tests should be done to improve the accuracy of diagnosis.

When one relates this prospective research study to clinical practice, it is apparent that a medical history and physical examination will be helpful in predicting patients with simple, clear-cut symptoms of exertional or stress incontinence to have incompetent urethra. When the symptoms are urge, mixed (stress and urge), or suggestive of overflow type, history and physical examination are poor predictors. The minimum study needed includes cystometry with residual urine check and provocative full-bladder stress testing. Cystometry will identify detrusor hyperactivity, while stress testing will assess urethral incompetence. The more complex urodynamic tests should be deferred to other unexplained cases.

The algorithmic method of Hilton and Stanton appears practical but has several weaknesses. ${ }^{10}$ This method proposes to evaluate incontinent patients first by eliminating urinary infection, gynecological abnormality, and fecal impaction. Once eliminated, the method relies heavily on the physician's ability to palpate a distended bladder. In our experience, this is a major feat to accomplish in obese patients and those who are only partially in retention. Once that patient is missed, she will be treated with antispasmodics if no stress incontinence is demonstrated on a provacative full-bladder stress test, which will further aggravate her condition. Another weakness of the algorithm is accepting an erroneous belief that all patients with stress incontinence will leak when stressed with a full bladder. Eastwood and Warrell modified the Hilton and Stanton algorithm by eliminating cystometry among patients who did not have a palpable bladder and were demonstrated to have stress incontinence with a full bladder. ${ }^{11}$ They proposed distinguishing mixed urethral incompetence and detrusor instability from pure sphincter incompetence based on the symptom only without cystometry. This modified algorithm successfully identified $80 \%$ of patients with pure detrusor instability, but only $25 \%$ of those with urethral incompetence or mixed detrusor instability and urethral incompetency.

It is, therefore, our conviction that, except for straightforward cases, incontinent patients should not be treated without documenting the cause, using the simplest, least invasive diagnostic procedure. We believe that a provocative full-bladder stress test and simple cystometry with residual urine measurement is safe, requires a minimum of expertise, and provides a high degree of accuracy and can be conducted in an office or bedside setting. The more sophisticated tests should be reserved for the complicated cases or for a research setting.

\section{ACKNOWLEDGMENT}

We thank Robert Wolfe, PhD, for assistance with statistical analysis and comments on manuscript drafts; Grace Lee Gillis, MA, Research Associate, for data management and analysis. In addition, we are deeply appreciative of all our competent project staff.

\section{REFERENCES}

1. Akhtar AJ, Broe GA, Crombie A, et al: Disability and dependence in the elderly at home. Age Ageing 2:102, 1973 
2. Yarnell JWG, Voyle GJ: The prevalence and severity of urinary incontinence in women. J Epidemiol Community Health 35:71, 1981

3. Diokno AC, Brock BM, Brown MB, et al: Prevalence of urinary incontinence and other urological symptoms in the noninstitutionalized elderly. J Urol 136: 1022, 1986

4. Thomas RM, Plymat KR, Blannin J, et al: Prevalence of urinary incontinence, Br Med J 281:1, 1980

5. Yarnell JWG, St. Leger AS: The prevalence, severity, and factors associated with urinary incontinence in a random sample of the elderly. Age Ageing 8:81, 1979

6. Wells $T$, Brink C, Diokno A: Urinary incontinence in elderly women: Clinical findings. J Am Geriatr Soc 35: 933-939, 1987

7. International Continence Society: First and second reports on standardization of terminology of lower urinary tract function. Urology 9:223, 1977
8. Abrams $P$, Feneley $R$, Torrens $M$ : Urodynamics. New York, Springer-Verlag, 1983, p 35

9. Castleden CM, Duffin HM, Asher MJ: Clinical and urodynamic studies in 100 elderly incontinent patients. Br J Urol 282:1103, 1981

10. Hilton P, Stanton SL: Algorithmic method for assessing urinary incontinence in elderly women. Br Med J 282:940, 1981

11. Eastwood $\mathrm{HDH}$, Warrell R: Urinary incontinence in the elderly female: Prediction in diagnosis and outcome of management. Age Ageing 13:230-234, 1984

12. Ouslander J, Hepps K, Raz $S$, et al: Genitourinary dysfunction in a geriatric outpatient population. J Am Geriatr Soc 34:507, 1986

13. Eastwood HDH: Urodynamic studies in the management of urinary incontinence in the elderly. Age Ageing 8:41, 1979 